\title{
Langmuir
}

pubs.acs.org/Langmuir

(C) 2009 American Chemical Society

\section{Influence of Cadmium and Selenate on the Interactions between Hormones and Phospholipids}

\author{
Barbara Gzyl-Malcher, ${ }^{* \dagger}$ Maria Filek, ${ }^{\ddagger}{ }^{\dagger}$ and Gerald Brezesinski \\ ${ }^{\dagger}$ Department of Physical Chemistry and Electrochemistry, Faculty of Chemistry, Jagiellonian University, \\ Ingardena 3, 30-060 Kraków, Poland, *Institute of Biology, Pedagogical University, Podbrzezie 3, \\ 31-054 Kraków, Poland, ${ }^{\S}$ Institute of Plant Physiology, Polish Academy of Sciences, Niezapominajek 21, \\ Krakow 30-239, Poland, and "Max Planck Institute of Colloids and Interfaces, Research Campus Golm, \\ D-14476 Potsdam, Germany
}

Received May 9, 2009. Revised Manuscript Received September 13, 2009

\begin{abstract}
In this study, the influence of plant hormones, negatively charged indolilo-3-acetic acid (IAA) and positively charged zeatin, on lipid membranes was studied. As models of negatively and positively charged biological membranes, monolayers of 1,2-dimyristoyl-sn-glycero-3-[phospho-L-serine] (DMPS) and 1,2-dipalmitoyl-3-trimethylammoniumpropane (DPTAP) at the water/air interface were used, respectively. Additionally, the effect of cadmium and selenium ions on the interactions between hormones and lipids was studied. Surface pressure and surface potential measurements, Brewster angle microscopy (BAM), and grazing incidence X-ray diffraction (GIXD) were used for that purpose. Both IAA and zeatin led to an expansion of the lipid monolayer caused by electrostatic interactions between oppositely charged groups: negatively charged polar group of DMPS and positively charged zeatin or positive DPTAP headgroup and negative IAA. The addition of ions to the subphase containing hormones causes competitive interactions of both solutes with oppositely charged lipid polar heads. The largest effect was observed for IAA. While zeatin does not change the domain shape of DMPS, IAA causes the complete disappearance of characteristic DPTAP domains. Addition of $\mathrm{SeO}_{4}{ }^{2-}$ ions causes restoration of DPTAP domains observed on pure water.
\end{abstract}

\section{Introduction}

Biological membranes generally contain mixtures of lipids that vary with respect to both their polar headgroups and their acyl chains. It has been admitted that the primary organizing force in the formation of these systems is dominated by the contribution of hydrophobic and hydrophilic hydration effects. ${ }^{1}$ The modification of membrane composition as a result of direct interaction with signal molecules present in the aqueous phase is a crucial factor for induction of many physiological processes in biological cells. Hormones are the group of substances essential for generating and controlling a wide range of responses in plants. It is now believed that some binding sites located on the plasmalemma play an essential role in the recognition of the information signals from the signaling molecules such as hormones. ${ }^{2,3}$ Unfortunately, the mechanisms by which hormones are recognized and through which their signal is transformed into the response are poorly understood. From our previous studies, performed in model systems based on natural lipid mixtures characteristic of embryoand nonembryogenic calli, it was concluded that hormones, especially auxins, can affect changes in head-headgroup distances $^{4-6}$ and electrokinetic potentials. ${ }^{7}$ It was also observed that processes of embryogenesis and regeneration were more effective

*Corresponding author. E-mail: gzyl@chemia.uj.edu.pl.

(1) Larson, C.; Moller, I. M.; Widell, S. The Plant Plasma Membrane; Springer: Berlin, 1990.

(2) Rubery, T. Annu. Rev. Plant Physiol. 1981, 32, 569.

(3) Libbenga, K. R.; Mennes, A. M. Hormone binding and signal transduction. In Plant Hormones: Physiology, Biochemistry and Molecular Biology; Davies, P. J., Ed.; Kluwer Academic Publishers: Dordrecht, 1995; pp 272-297.

(4) Laggner, P.; Filek, M.; Marcińska, I; Szechyńska-Hebda, M; Kriechbaum, M. Plant Sci. 2003, 165, 265.

(5) Filek, M.; Gzyl, B.; Dudek, A. Cell. Mol. Biol. Lett. 2003, 8, 713.

(6) Gzyl, B.; Filek, M.; Dudek, A. J. Colloid Interface Sci. 2004, 269, 153.

(7) Filek, M.; Zembala, M.; Szechyńska-Hebda, M. Z. Naturforsch. 2002, 57C, in the presence of small amounts of some ions, especially selenium. ${ }^{8}$ However, higher concentrations of this substance as well as other metal ions lead to the destruction of cells. ${ }^{9,10}$

A variety of toxic contaminants, including cadmium and selenium, are a result of increased environmental burdens from industrial, agricultural, energy, and municipal sources. ${ }^{11-13}$ Plants can absorb and accumulate a large amount of $\mathrm{Cd}$ and $\mathrm{Se}$ in their tissues, and thus, they are transmitted to other organisms. At least $70 \%$ of the Cd intake by humans originates from plant foods. ${ }^{14}$ Selenium hyperaccumulation occurs mainly in naturally seleniferous soils and causes disease and death in animals. ${ }^{15,16}$ The question about how $\mathrm{Cd}$ and Se act at the cellular level and how cells may defend themselves against these pollutants is receiving increasing attention. ${ }^{13}$ To understand the mode of action leading to heavy metal function in living cells, physicochemical properties of lipid monolayers (as a model of the biological membrane) can serve as the source of many theoretical data concerning the structural and mechanical properties of the molecules building these layers. ${ }^{1,17}$

(8) Stadtman, T. C. Annu. Rev. Biochem. 1990, 59, 111.

(9) Anderson, J. W. Selenium interactions in sulfur metabolism. In Sulfur Nutrition and Assimilation in Higher Plants - Regulatory, Agricultural and Environmental Aspects; De Kok, L. J., Ed.; SPB. Academic Pub.: The Hague, 1993; pp 49-60.

(10) Galloway, J. N.; Thornton, J. D.; Norton, S. A.; Volcho, H. L.; McLean, R. A. Atmos. Environ. 1982, 16, 1677.

(11) Adriano, D. C. Trace Element in the Terrestrial Environment; SpringerVerlag: New York, 1986; pp 15-25.

(12) Angelone, M.; Bini, C. Trace elements concentrations in soils and plants of Western Europe. In Biogeochemistry of Trace Metals; Adriano, D. C., Ed.; Lewis Publishers: Boca Raton, FL, 1992; pp 19-60.

(13) Schützendübel, A.; Polle, A. J. Exp. Bot. 2002, 53, 1351.

(14) Wagner, G. J. Adv. Agron. 1993, 51, 173

(15) Feist, L. J.; Parker, D. R. New Phytol. 2001, 149, 61.

(16) Rosenfeld, I.; Beath, O. A. Selenium, Geobotany, Biochemistry, Toxicity and Nutrition; Acc. Press: New York, 1964

(17) Gzyl, B.; Paluch, M. Prog. Colloid Polym. Sci. 2004, 126, 60. 
The model membranes are characterized by specific hydration levels and a specific distribution of water and water-soluble substances. ${ }^{18}$

Thus, the aim of this study was to describe the surface properties of lipid model membranes in the presence of $\mathrm{Cd}^{2+}$ and $\mathrm{SeO}_{4}{ }^{2-}$ ions with selected hormones. As a model of negatively charged biological membranes, DMPS was chosen. DPTAP was mimicking the positive parts in membranes. IAA, representing a group of negatively charged hormones (auxins), and zeatin, representing positively charged hormones, were dissolved in the water subphase. The effect of cadmium and selenium ions on the interactions between hormones and lipids was studied by $\pi-A$ isotherms, in combination with Brewster angle microscopy (BAM) and surface potential measurements. Both methods provide characterization of monolayers at different length scales and are based on differences between a bare water surface and a monolayer-covered surface.

\section{Experimental Section}

Materials. Indolilo-3-acetic acid (IAA), 6-(4-hydroxy-3methylbut-2-enylamino)purine (zeatin), cadmium dichloride, and sodium selenate ( $>98 \%$ pure) were obtained from Sigma Aldrich Company (St. Louis, MO). The following compounds ( $>99 \%$ pure) were purchased from Avanti Polar Lipids (Alabaster, AL): 1,2-dimyristoyl-sn-glycero-3-[phospho-L-serine] (DMPS) and 1,2-dipalmitoyl-3-trimethylammonium-propane (DPTAP). Chloroform (Merck, Germany) was the spreading solvent. The subphase for all experiments was water first distilled and then purified by a Millipore system with a specific resistance above $18 \mathrm{M} \Omega \cdot \mathrm{cm}$.

Methods. Film Balance and Surface Potential Measurements. Surface pressure-area isotherms ( $\pi-A$ isotherms) were measured on a homemade Teflon trough. The surface pressure was recorded with a continuous Wilhelmy-type pressure measuring system using filter paper as Wilhelmy plate with an accuracy better than $0.1 \mathrm{mN} \cdot \mathrm{m}^{-1}$. The substances were spread from $1 \mathrm{mM}$ chloroform solutions onto the water surface using a $100 \mu \mathrm{L}$ syringe. The monolayers were compressed at a rate of $5 \AA^{2} \cdot$ molecule ${ }^{-1} \cdot \mathrm{min}^{-1}$ after allowing about $10 \mathrm{~min}$ for evaporation of the solvent. The concentration of hormones was $1 \mu \mathrm{M}$, chosen on the basis of earlier experiments. The salts were used in concentration inducing heavy metal stress in natural plant cells in in vitro wheat cultures, that is, $5 \mathrm{mM}$ (data in preparation).

Surface potential-area $(\Delta V-\mathrm{A})$ isotherms were recorded using the above-described trough. During monolayer compression, $\pi$ and $\Delta V$ were recorded simultaneously. The surface potential sensor consists of a vibrating electrode (constructed by V. L. Shapovalov, N. N. Semenov Institute of Chemical Physics RAS, Moscow, Russia), which is suspended above the monolayer. The isotherms were measured several times and have a good reproducibility.

Brewster Angle Microscopy. The morphology of the monolayers was visualized using the Brewster angle microscope (BAM1 from NFT Göttingen, Germany). The resolution is about $2 \mu \mathrm{m}$. The optical system and the Langmuir film balance are mounted on an antivibration table. A $p$-polarized beam of a diode laser is reflected at a mirror and strikes the water surface under the Brewster angle to give zero reflection. ${ }^{19,20}$ The presence of a monolayer alters the optical properties of the interface, resulting in enhanced reflectivity in film-covered regions, and thus permits domains to be visualized with an appropriate detector. Monolayer compression is recorded on videotape, and frames are grabbed and digitized by a computer.

(18) Gzyl-Malcher, B.; Handzlik, J.; Nowak-Stepniowska, A. Colloids Surf., A 2008, 321, 52 .

(19) Hönig, D.; Möbius, D. J. Phys. Chem. 1991, 95, 4590

(20) Hénon, S.; Meunier, J. Rev. Sci. Instrum. 1991, 62, 936.

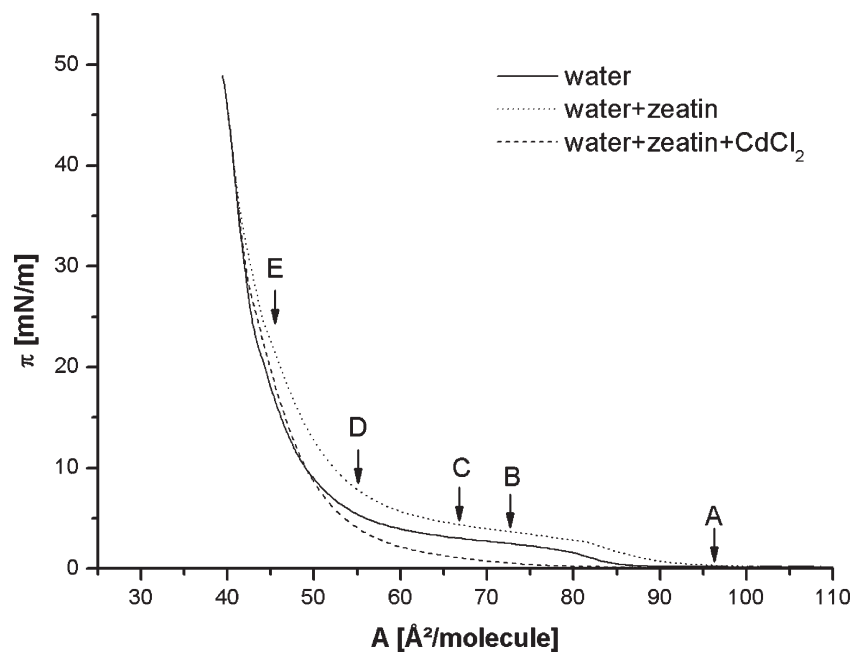

Figure 1. Surface pressure versus mean molecular area isotherms of DMPS monolayer on water, water with zeatin, and water with zeatin and cadmium dichloride.

Grazing Incidence X-ray Diffraction. Grazing incidence X-ray diffraction (GIXD) experiments were performed at the undulator beamline BW1 at HASYLAB, DESY (Hamburg, Germany) using the liquid-surface diffractometer. The incident angle, $\alpha_{i}$, of the monochromatic beam $(\lambda \approx 1.3 \AA)$ was kept below $\left(\alpha_{i}=0.85 \alpha_{c}\right)$ the critical angle of total reflection for the air/water interface, $\alpha_{c}=0.13^{\circ}$, and the diffracted intensity was recorded as a function of the horizontal and vertical scattering angles $2 \theta_{x y}$ and $\alpha_{\mathrm{f}}$, respectively, by a linear position sensitive detector (OEM100-M, Braun, Garching, Germany). A Soller collimator giving a horizontal resolution of $0.008 \AA^{-1}$ was placed in front of the detector, and the entire system (collimator and detector) was rotated to set the horizontal scattering angle. The out-of-plane $\left(Q_{z} \approx(2 \pi / \lambda) \sin \left(\alpha_{\mathrm{f}}\right)\right)$ and in-plane $\left(\mathrm{Q}_{x y} \approx(4 \pi / \lambda) \sin \left(2 \theta_{x y} / 2\right)\right)$ components of the scattering vector $\mathbf{Q}$ provide information about the laterally periodic structures of the monolayer in terms of lattice parameters, tilt angle, tilt direction, and lattice distortion. Detailed descriptions of the experimental setup and the theoretical background of the GIXD experiment can be found in the literature. ${ }^{21-26}$ The tilt angles of the aliphatic chains have error bars below $\pm 1^{\circ}$.

\section{Results}

The surface pressure-area isotherm of DMPS spread at the air-water interface at $20^{\circ} \mathrm{C}$ is shown in Figure 1 . The isotherm is in good agreement with the reports of other authors. ${ }^{27}$ The isotherm shows a plateau region where the surface pressure varies very slowly with area. Such drastic changes in the slope of the isotherm are interpreted as phase transition from the liquid expanded (LE) to a liquid condensed (LC) phase in the monolayer. The transition into the $\mathrm{LC}$ phase starts at $1.8 \mathrm{mN} \cdot \mathrm{m}^{-1}$. The presence of zeatin in the subphase has a clear expanding effect.

(21) Jacquemain, D.; Leveiller, F.; Weinbach, S. P.; Lahav, M.; Leiserowitz, L.; Kjaer, K.; Als-Nielsen, J. J. Am. Chem. Soc. 1991, 113, 7684

(22) Kjaer, K. Phys. B 1994, 198, 100.

(23) Als-Nielsen, J.; Jacquemain, D.; Kjaer, K.; Leveiller, F.; Lahav, M.; Leiserowitz, L. Phys. Rep. 1994, 246, 251.

(24) Brezesinski, G.; Dietrich, A.; Struth, B.; Böhm, C.; Bouwman, W. G.; Kjaer, K.; Möhwald, H. Chem. Phys. Lipids 1995, 76, 145.

(25) Jensen, T. R.; Kjaer, K. Structural properties and interactions of thin films at the air-liquid interface explored by synchrotron X-ray scattering. In Novel Methods to Study Interfacial Layers, Studies in Interface Science; Moebius, D., Miller, R., Eds.; Elsevier Sciences: Amsterdam, 2001; Vol. 11, pp 205-254.

(26) Kaganer, V. M.; Brezesinski, G.; Möhwald, H.; Howes, P. B.; Kjaer, K. Phys. Rev. Lett. 1998, 81, 5864.

(27) Letellier, S. R.; Lochhead, M. J.; Campbell, A. A.; Vogel, V. Biochim. Biophys. Acta 1998, 1380, 31 
Additionally, the phase transition pressure is increased to $2.6 \mathrm{mN} \cdot \mathrm{m}^{-1}$. When $\mathrm{CdCl}_{2}$ was added to the subphase (water with zeatin), a condensation of the DMPS monolayer is observed: The plateau pressure is diminished to $0.3 \mathrm{mN} \cdot \mathrm{m}^{-1}$. However, both zeatin and cadmium ions do not influence the limiting area value observed for DMPS monolayer on pure water at high pressure.

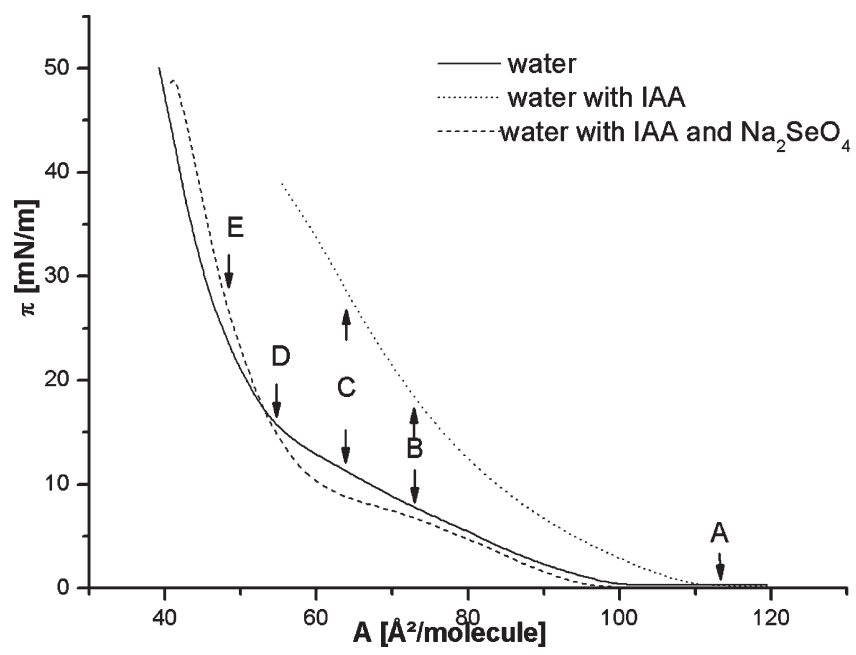

Figure 2. Surface pressure versus mean molecular area isotherms of DPTAP monolayer on water, water with IAA, and water with IAA and natrium selenate.
The $\pi-A$ isotherm of DPTAP is presented in Figure 2. The transition from the liquid-expanded to a condensed phase starts at around $8 \mathrm{mN} \cdot \mathrm{m}^{-1}$. The plateau region is far from being horizontal. Obviously, the transition into the condensed phase is not very cooperative. The addition of organic and inorganic substances to the subphase induces the same type of changes as observed for the DMPS monolayer. The presence of IAA shifts the $\pi-A$ isotherm to much larger molecular areas $\left(A_{\text {lim }}\right.$ extrapolated to zero pressure is $53.6 \AA^{2} \cdot$ molecule $^{-1}$ for pure water and $86.2 \AA^{2} \cdot$ molecule $^{-1}$ for water with IAA). The monolayer is more expanded, and the $\mathrm{LE} / \mathrm{LC}$ transition disappears. The addition of salt $\left(\mathrm{Na}_{2} \mathrm{SeO}_{4}\right)$ to the subphase (water with IAA) causes a reduction of the molecular area in the liquid-expanded state and shifts the transition pressure to $6.6 \mathrm{mN} \cdot \mathrm{m}^{-1}$. The two-phase coexistence region is now better pronounced. The limiting area in the condensed state is slightly larger compared with that on water.

Brewster angle micrographs of DMPS are displayed in Figure 3, for areas per molecule marked by arrows in Figure 1. While a homogeneous fluid film was observed at the lowest surface pressures (A), bright domains appear during film compression at the onset of the plateau in the corresponding isotherms (B). These domains represent a condensed phase surrounded by the liquid-expanded phase within the two-phase coexistence region. With decreasing area per molecule, the domains grow and form several arms (C). Finally, during further compression, they tend to fuse $(\mathrm{D}, \mathrm{E})$. The overall impression from Figure 3 is that the shapes of the domains are not very sensitive to the presence of salt or/and hormone in the subphase. However, the size of condensed

a)

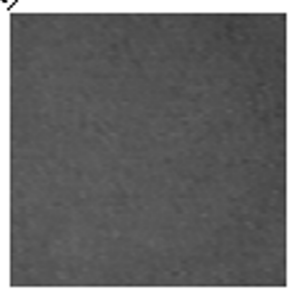

A

b)

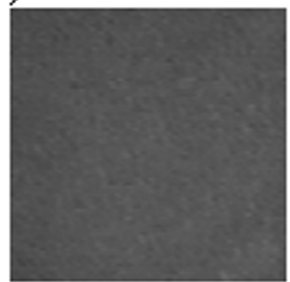

A

c)

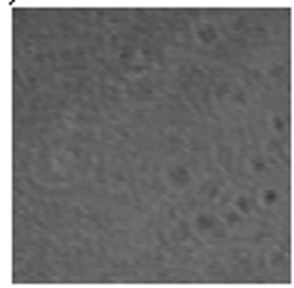

A

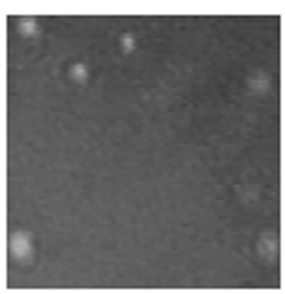

B

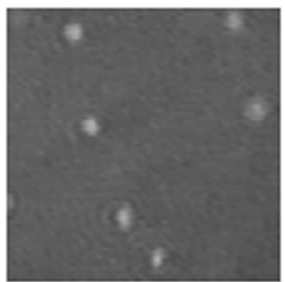

B

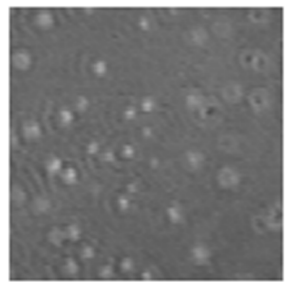

B

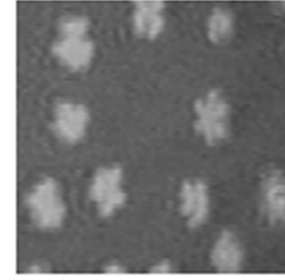

C

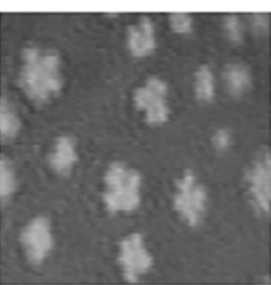

C

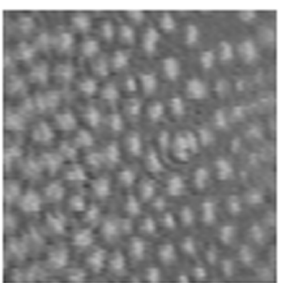

C

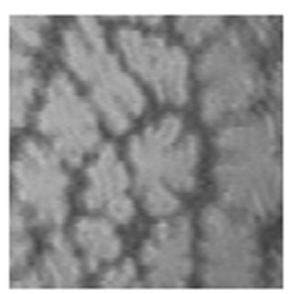

$\mathrm{D}$

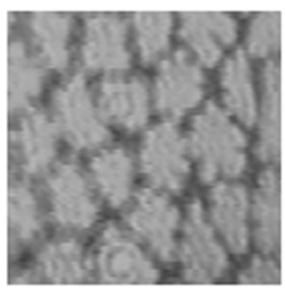

$\mathrm{D}$

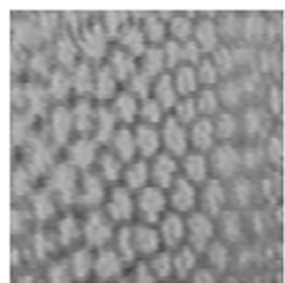

$\mathrm{D}$

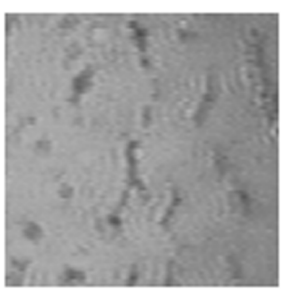

E

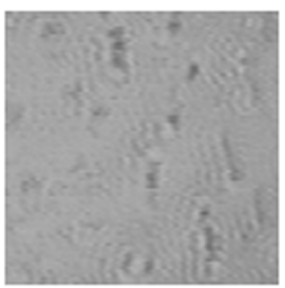

E

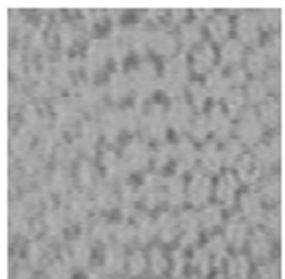

E

Figure 3. Brewster angle micrographs of DMPS monolayer on (a) water, (b) water with zeatin, and (c) water with zeatin and cadmium dichloride. 
a)

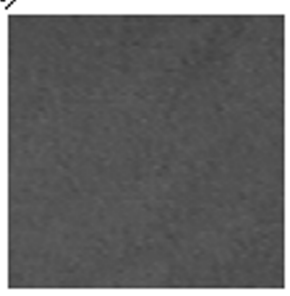

A

b)

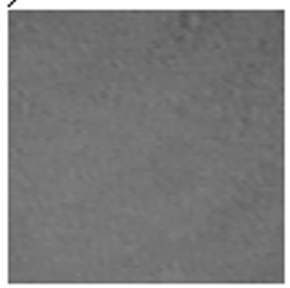

A

c)

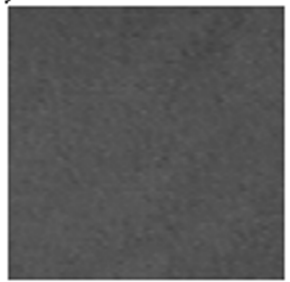

A

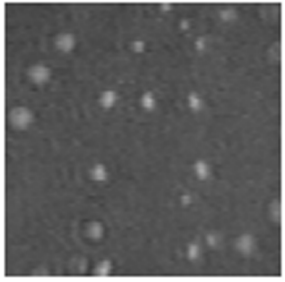

B

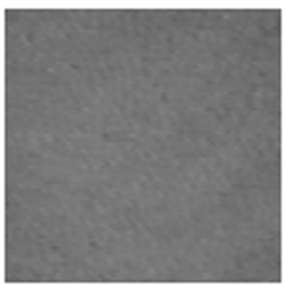

B

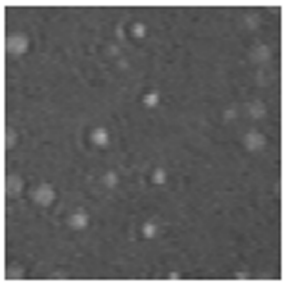

B

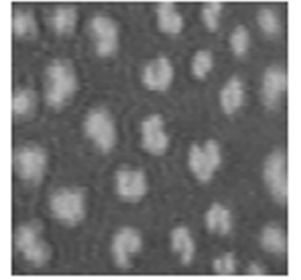

C

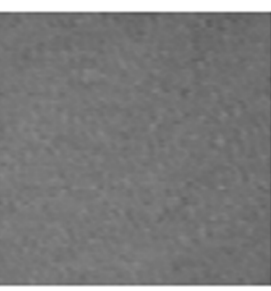

C

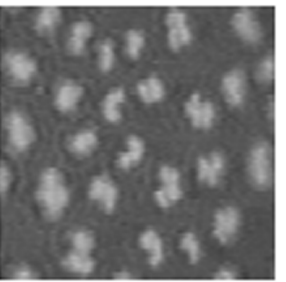

C

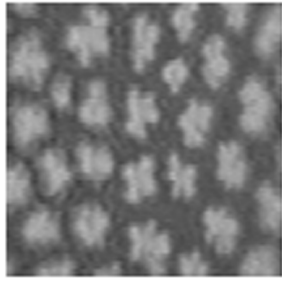

D

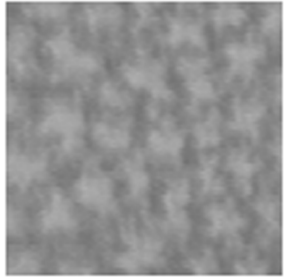

E

Figure 4. Brewster Angle Micrographs of DPTAP monolayer on (a) water, (b) water with IAA, and (c) water with IAA and natrium selenate.

domains is strongly dependent on the type of solutes in water. Domains similar to those on pure water (Figure 3a) can be observed in the case of zeatin (Figure 3b), whereas the addition of salt reduces the size and increases the number of the LC domains drastically (Figure 3c).

Figure 4 shows BAM images for DPTAP on different subphases taken at molecular areas marked by arrows in Figure 2. In the case of water (Figure 4a), one can observe the appearance of LC domains, their growing in number and size, and finally coalescence with increasing surface pressure. When IAA is added to the subphase, no domains of condensed DPTAP are observed (Figure 4b). However, domains with similar shape and size compared with those observed on pure water can be seen in the presence of salt (Figure 4c). The disappearance of domains on the IAA containing subphase is in agreement with the expanded isotherm.

GIXD experiments were performed on water, on the IAA, as well as on the IAA and $\mathrm{Na}_{2} \mathrm{SeO}_{4}$ containing subphases to determine the monolayer structure in the angstrom range. Figure 5 shows selected contour plots of the corrected X-ray intensities as a function of the in-plane and the out-of-plane scattering vector components. On water, DPTAP exhibits an unusual phase sequence: orthorhombic with $\mathrm{NN}$ (nearest neighbor) tilt $\left(\mathrm{L}_{2}\right.$ phase)-oblique-orthorhombic with NNN (next nearest neighbor) tilt (Ov phase) on compression. This shows that the tilt direction does not change at a defined transition pressure (second order phase transition) as observed for fatty acids and fatty acid esters, ${ }^{28}$ but there is a continuous transition

(28) Kaganer, V. M.; Möhwald, H.; Dutta, P. Rev. Mod. Phys. 1999, 71, 779.

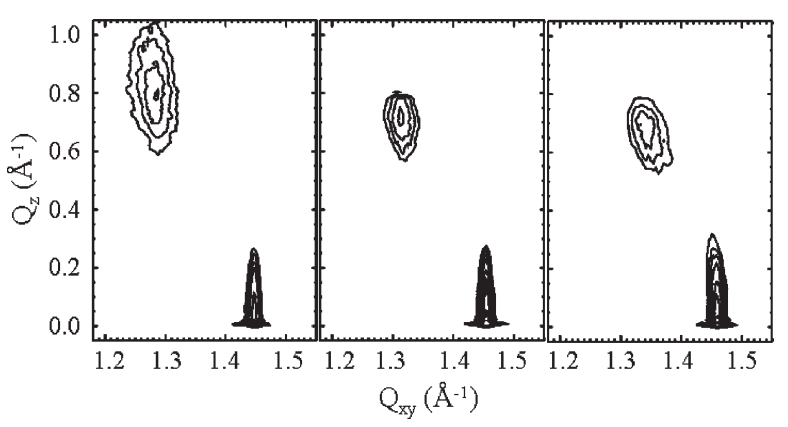

Figure 5. Selected contour plots of the corrected X-ray intensities as a function of the in-plane and out-of-plane scattering vector components $Q_{x y}$ and $Q_{z}$, respectively, of DPTAP on a subphase containing IAA and $\mathrm{Na}_{2} \mathrm{SeO}_{4}$. The results obtained at 15, 25, and $35 \mathrm{mN} \cdot \mathrm{m}^{-1}$ (from left to right) are shown.

from $\mathrm{NN}$ ( $\mathrm{L}_{2}$ phase) to $\mathrm{NNN}$ (Ov phase) tilt over a certain pressure range. On the subphase containing IAA and $5 \mathrm{mM} \mathrm{Na}_{2} \mathrm{SeO}_{4}$, the intensity distribution with two Bragg peaks (one at zero $Q_{z}$ and the second one at $Q_{z}>0$ ) shows only the $\mathrm{L}_{2}$ phase (orthorhombic unit cell with NN tilted molecules) at all pressures investigated. The tilt angle is drastically increased (Figure 6). This is obviously the reason for the missing transition to the Ov phase, which occurs at smaller tilt angles. Plotting $1 / \cos (t)$ versus the lateral pressure gives a linear relation. ${ }^{29}$ The extrapolation to zero tilt allows the determination of the transition pressure to the nontilted state. This nontilted state can

(29) Bringezu, F.; Dobner, B.; Brezesinski, G. Chem.-Eur. J. 2002, 8, 3203. 


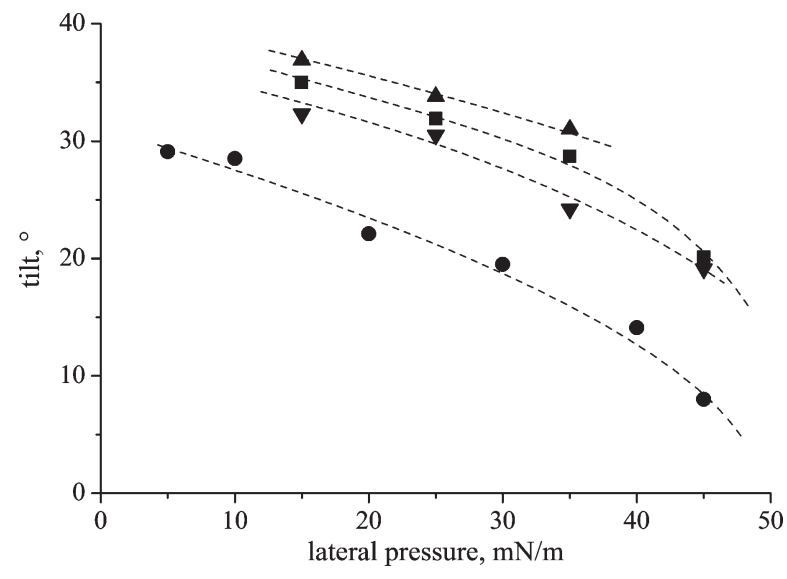

Figure 6. Tilt angles of the aliphatic chains of (๑) DPTAP on water, $(\boldsymbol{\Delta})$ on a subphase containing IAA and $\mathrm{Na}_{2} \mathrm{SeO}_{4},(\boldsymbol{\nabla})$ on $5 \mathrm{mM} \mathrm{NaCl}$, and (ם) on $5 \mathrm{mM} \mathrm{Na}_{2} \mathrm{SeO}_{4}$ as a function of the lateral pressure. Note that DPTAP does not form a condensed monolayer on the IAA containing subphase.

be reached on water at $48 \mathrm{mN} \cdot \mathrm{m}^{-1}$, whereas the presence of IAA and $\mathrm{Na}_{2} \mathrm{SeO}_{4}$ in the subphase shifts the transition to $74 \mathrm{mN} \cdot \mathrm{m}^{-1}$. The cross-sectional area of the lipid chains is also slightly increased: $20.2 \AA^{2}$ on water and $20.5 \AA^{2}$ on the hormone/salt solution. The observed increase of the tilt angle leads to a larger in-plane area of DPTAP on the hormone/salt solution compared with water, what is in perfect agreement with the observed shift in the isotherm. GIXD experiments of DPTAP on the IAA solution did not show any Bragg peaks indicating the disordered state of the monolayer in correspondence with the expanded isotherm. Additionally, DPTAP has been measured on $5 \mathrm{mM}$ salt solutions $\left(\mathrm{Na}_{2} \mathrm{SeO}_{4}\right.$ or $\left.\mathrm{NaCl}\right)$ for comparison. In both cases, the same phase behavior as on the salt/hormone solution has been observed; that is, only the $\mathrm{L}_{2}$ phase can be seen in the whole pressure region. The surprising observation is that the different anions influence the DPTAP monolayer structure in a slightly different way. Previous experiments have shown that a critical charge density is essential to observe size effects of ions participating in the electrical double layer. ${ }^{30,31}$ The tilt of the chains increases in both cases compared with the structure on water. The effect is larger for the selenate counterion compared with the chloride anion (Figure 6).

Figure 7 shows the surface potential measurements $(\Delta V)$ for DMPS monolayers on different subphases. The $\Delta V-A$ isotherm in the absence of any solutes in water demonstrates a sharp rise of $\Delta V$ near $100 \AA^{2} \cdot$ molecule $^{-1}$, which is approximately the molecular area at which the pressure in the liquid-expanded phase starts to increase, followed by a slow increase during further compression until $\Delta V=170 \mathrm{mV}$. At that point, which corresponds approximately to the end of the LE/LC phase transition region, the slope of the $\Delta V-A$ isotherm is changing and the surface potential rises more rapidly until $300 \mathrm{mV}$. The presence of zeatin in the subphase does not cause any change in the surface potential. Addition of cadmium causes an increase in $\Delta V$ values (by $\sim 200 \mathrm{mV}$ ), but the slope of the $\Delta V-A$ isotherm is almost unchanged.

DPTAP on water (Figure 8) shows similar behavior of surface potential as that described for DMPS. One can distinguish a sharp increase in $\Delta V$ from 360 to $610 \mathrm{mV}$, a slow rise until $720 \mathrm{mV}$, and a change in slope of the $\Delta V-A$ isotherm leading to a faster increase until $1040 \mathrm{mV}$. When IAA is injected into the subphase, the surface

(30) Shapovalov, V. L.; Brezesinski, G. J. Phys. Chem. B 2006, 110, 10032.

(31) Shapovalov, V. L.; Ryskin, M. E.; Konovalov, O. V.; Hermelink, A.; Brezesinski, G. J. Phys. Chem. B 2007, 111, 3927.

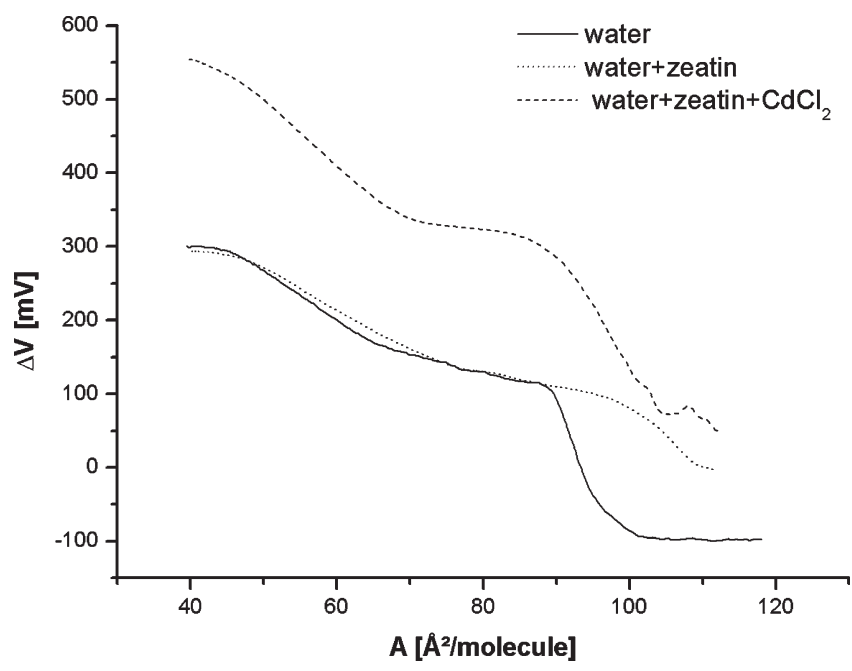

Figure 7. Surface potential versus mean molecular area isotherms of DMPS monolayer on water, water with zeatin, and water with zeatin and cadmium dichloride.

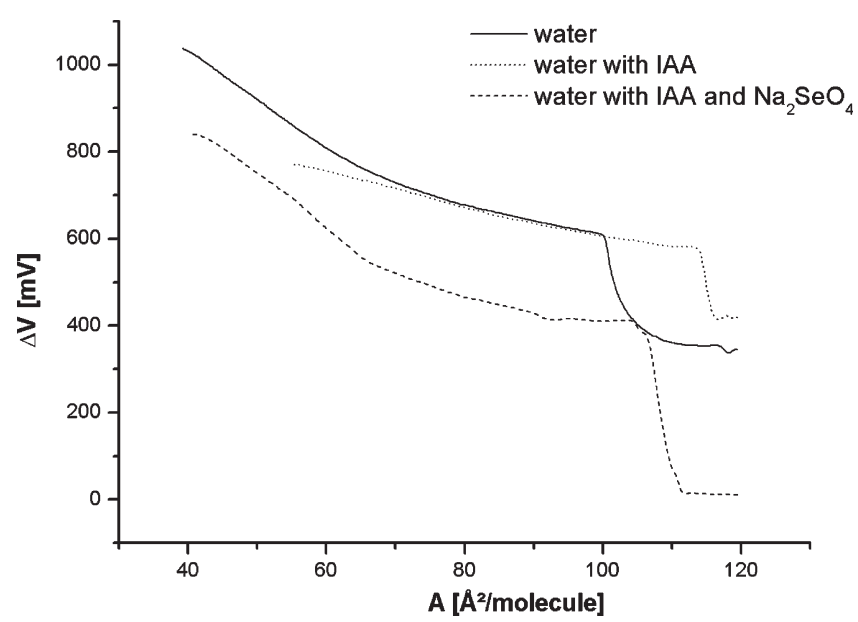

Figure 8. Surface potential versus mean molecular area isotherms of DPTAP monolayer on water, water with IAA, and water with IAA and natrium selenate.

potential values of DPTAP are very close to those on pure water. However, that $\Delta V-A$ isotherm, unlike the others, is characterized by a constant slope, indicating that there is no change in the phase state of the monolayer. The addition of $\mathrm{SeO}_{4}{ }^{2-}$ and IAA decreases the surface potential values (by $\sim 170 \mathrm{mV}$ ) and restores the slopes (corresponding to the observed phase transitions) measured for the DPTAP monolayer on pure water.

\section{Discussion}

In this study, the surface pressure-molecular area measurements supplemented with BAM, surface potential and GIXD experiments offer a unique set of tools for monitoring the interaction of hormones and ions with model membranes.

From the $\pi-A$ isotherms it is evident that both hormones, zeatin and IAA, led to an expansion of the lipid monolayer. Such an expansion effect, induced by hormones present in the water phase, was also observed for monolayers of phospholipids extracted from natural plant membranes. ${ }^{32}$ As a result of electrostatic interactions between oppositely charged groups

(32) Gzyl, B.; Filek, M.; Dudek, A. Z. Naturforsch. 2003, 59C, 60. 
(negatively charged polar group of DMPS and positively charged zeatin or positive DPTAP headgroup and negative IAA), hormones penetrate into the fluid monolayers. The expansion effect of zeatin is much smaller compared with that of IAA. Zeatin expands the fluid state of the monolayer and shifts the LE/LC transition pressure to slightly higher values. Upon compression, it is squeezed out from the condensed monolayer at high pressure indicated by the same molecular area of DMPS on the different subphases. In contrast, IAA interaction with DPTAP leads to the complete suppression of the condensed phase. Both BAM and GIXD experiments show that there is no transition into a condensed phase. While zeatin does not change the domain shape of DMPS, IAA causes the complete disappearance of characteristic DPTAP domains. The $\Delta V-A$ isotherms are also almost not influenced by the hormones. In the case of zeatin, the same change in slope connected with the LE/LC phase transition has been observed. The $\Delta V-A$ isotherm of DPTAP on IAA is similar to the one on water until the phase transition into the condensed phase. At higher pressure, the $\Delta V$ values of the disordered DPTAP on IAA are smaller compared with the ordered DPTAP on water. The large effect of IAA on the structural properties of model lipid membranes was also observed by X-ray experiments in bulk systems. ${ }^{4}$

The addition of ions to the subphase containing hormones causes competitive interactions of both solutes with oppositely charged lipid polar heads. It is possible that divalent ions bridge adjacent lipid molecules, promoting monolayer condensation, what is easily seen from the $\pi-A$ isotherms. The LE/LC phase transition pressure is decreased in both cases. The condensed part of the DMPS isotherm is almost not influenced if $\mathrm{Cd}^{2+}$ ions are present together with the zeatin, whereas condensed DPTAP is shifted to larger molecular areas upon interaction with $\mathrm{SeO}_{4}{ }^{2-}$ ions in the presence of IAA. This expanding effect is the result of the increased effective area of the headgroup seen by the measured increase of the tilt angle of the aliphatic chains of DPTAP. The phase sequence on the subphase containing IAA and $\mathrm{SeO}_{4}{ }^{2-}$ ions is also changed compared to that on water. The tilt angle is much larger and does not reach the value of approximately $15^{\circ}$ which seems to be crucial for the formation of the Ov phase (orthorhombic packing of NNN tilted chains) in the DPTAP monolayer on water. It is worth mentioning that the unusual continuous transition from the $\mathrm{L}_{2}$ to the $\mathrm{Ov}$ phase via an oblique phase has been previously observed only in binary mixtures. ${ }^{33}$ The GIXD experiments using different anions $\left(\mathrm{SeO}_{4}{ }^{2-}\right.$ or $\mathrm{Cl}^{-}$) showed an unexpected ion size effect. ${ }^{30,31}$ The larger anion induces a larger tilt of the aliphatic chains of DPTAP. This indicates that the area requirement of the headgroup increases compared with that on the pure water subphase. The charge of the headgroup is not influenced, but obviously it is not the only reason (electrostatic repulsion between like charges) for the large area requirement. The bulky trimethyl group contributes to a certain extent to this area requirement. The increase of the tilt angle cannot be explained by screening the electrostatic repulsion as may be expected. The counterions interact strongly with the cationic headgroup and penetrate into the headgroup region, leading to the observed increase of the headgroup area. The larger

(33) Krasteva, N.; Vollhardt, D.; Brezesinski, G. J. Phys. Chem. B 2000, 104, 8704 anion has the larger effect. Taking this into account, the observed further increase of the tilt angle on the IAA/ $\mathrm{Na}_{2} \mathrm{SeO}_{4}$ subphase shows that there is a competition between the counterions and the hormones for the interaction with the cationic headgroup. However, IAA is not completely replaced by $\mathrm{SeO}_{4}{ }^{2-}$ but is still present to a small extent in the headgroup region leading to the further tilt angle increase.

Ions introduced into the subphase cause also large changes in the surface potential, in the order of $200 \mathrm{mV}$, by forming the electric double layer around the charged headgroups. Such a big increase or decrease in surface potential might cause membrane destruction under natural conditions. In natural cells, different stress factors (including chemical substances) induce depolarization and/or hyperpolarization of membrane potential. ${ }^{34-36} \mathrm{Such}$ electric changes serve as messenger in cell signal transduction. High increase or decrease of surface potential induced by $\mathrm{SeO}_{4}{ }^{2-}$ and $\mathrm{Cd}^{2+}$ ions confirm the stress character of these ions on cell membranes. The outer plant cell membrane (plasmalemma) is built mainly by phospholipids and proteins and contains negatively and positively charged centers. The strong binding of $\mathrm{SeO}_{4}{ }^{2-}$ and $\mathrm{Cd}^{2+}$ ions to these centers may be responsible for the maintenance of membrane potential on higher/lower levels in comparison with the resting membrane potential. That effect can cause stress observed in natural cells under the influence of ions in concentrations used in our experiments (data in preparation). Competition in interactions of zeatin and $\mathrm{Cd}^{2+}$ with negatively charged monolayers leads to the decreased size of condensed domains observed by BAM. Bridging of adjacent lipid molecules by the $\mathrm{Cd}^{2+}$ ions decreases the distances between molecules in the monolayer and increases the nucleation rate, and as a result many small condensed domains can be observed. In the case of a positively charged monolayer, the presence of ions $\left(\mathrm{SeO}_{4}{ }^{2-}\right)$ causes restoration of domains observed on pure water. IAA has a strong fluidizing effect on the positively charged DPTAP monolayer, which is reduced by the $\mathrm{SeO}_{4}{ }^{2-}$ ions. Therefore, the competitive interaction of inorganic ions and organic hormones is a predominant factor to suppress the influence of organic ions.

\section{Concluding Remarks}

In our model system, where DMPS and DPTAP monolayers represent negatively and positively charged centers of membranes, respectively, cadmium and selenate ions modify the interactions of hormones with these centers. Probably, the presence of ions hinders the penetration of the hormones into the membrane. That is especially important in the case of positively charged centers, where negatively charged solutes significantly modify the membrane structure. As regards negatively charged centers, positively charged ions induce structural changes in spite of hormone presence in the system.

Acknowledgment. This work was partly supported by Grant KBN 1 T09A 122 30. We thank HASYLAB at DESY, Hamburg, Germany, for beamtime and excellent support.

(34) Fromm, J.; Eschrich, W. J. Plant Physiol. 1993, 141, 673

(35) Filek, M.; Kościelniak, J. Plant Sci. 1997, 123, 39.

(36) Dziubińska, H.; Filek, M.; Kościelniak, J.; Trebacz, K. J. Plant Physiol. 2003, 160, 1203 . 